\title{
Demixing in binary mixtures of apolar and dipolar hard spheres
}

\author{
N. G. Almarza, ${ }^{\text {a) }}$ E. Lomba, C. Martín, and A. Gallardo \\ Instituto de Química Física Rocasolano, CSIC, Serrano 119, E-28006 Madrid, Spain \\ (Received 15 September 2008; accepted 8 November 2008; published online 16 December 2008)
}

\begin{abstract}
We study the demixing transition of mixtures of equal size hard spheres and dipolar hard spheres using computer simulation and integral equation theories. Calculations are carried out at constant pressure, and it is found that there is a strong correlation between the total density and the composition. The critical temperature and the critical total density are found to increase with pressure. The critical mole fraction of the dipolar component on the contrary decreases as pressure is augmented. These qualitative trends are reproduced by the theoretical approaches that on the other hand overestimate by far the value of the critical temperature. Interestingly, the critical parameters for the liquid-vapor equilibrium extrapolated from the mixture results in the limit of vanishing neutral hard sphere concentration agree rather well with recent estimates based on the extrapolation of charged hard dumbbell phase equilibria when dumbbell elongation shrinks to zero [G. Ganzenmüller and P. J. Camp, J. Chem. Phys. 126, 191104 (2007)]. (C) 2008 American Institute of Physics. [DOI: 10.1063/1.3039512]
\end{abstract}

\section{INTRODUCTION}

In the past years a considerable research effort has been devoted to the study of phase equilibria of dipolar fluids using both computer simulation techniques ${ }^{1-9}$ and theory. ${ }^{10,11}$ In a first stage, it was commonly assumed that some dispersion interaction should be present for liquid-vapor equilibrium (LVE) to exist. ${ }^{4,12}$ Recently, however, new computer simulation results $8,9,13,14$ seem to have reopened the discussion regarding the existence of LVE. Ganzenmüller and Camp $^{13}$ estimated a probable location of the hypothetical LVE critical point of the dipolar hard sphere (DHS) system. These authors utilized an extrapolation of the LVE results for charged hard dumbbells. Such an extrapolation is needed since the computational effort required to reach the DHS limit is prohibitive. Using an alternative approach, Pshenichnikov and Mekhonoshin ${ }^{14}$ found also some evidence of a gas-liquid transition using Monte Carlo simulation of DHS under confinement.

Related systems composed of hard and soft dipolar spheres have been studied by Hynninen and Dijkstra. ${ }^{15,16}$ In their work the dipole moments are constrained to lie parallel to an external field. Under these conditions no stable LVE was found.

Lately, the LVE of Stockmayer fluids (whose particles interact through a Lennard-Jones potential in addition to the dipole-dipole term) has also been extensively reviewed using computer simulation. In the limit of high reduced dipole moments it is expected that the dipolar interactions should play the key role in a possible phase separation. However, once more, the controversy regarding the system behavior in such a limit is far from settled. ${ }^{17-19}$

The fluid phase behavior of mixtures of DHSs has been studied using integral equation theory (IET) and density functional theory (DFT). Chen et al. ${ }^{20}$ used the reference

${ }^{a)}$ Electronic mail: noe@iqfr.csic.es. hypernetted chain (RHNC) approximation to analyze the demixing phase transition in mixtures of apolar hard spheres (HSs) and DHS. Range and Klapp ${ }^{21}$ studied the phase behavior of binary mixtures of equisized DHS with different dipole moments in the fluid phase regime using DFT. These authors predicted the existence of a first order transition between an isotropic gas and a ferroelectric fluid, with the first order transition ending in a tricritical point. In a related work, Szalai and Dietrich ${ }^{22}$ applied a mean field DFT to Stockmayer fluid mixtures.

Concerning simulation results, De Leeuw et al. ${ }^{23}$ studied the phase behavior of mixtures of Stockmayer and LennardJones molecules using molecular dynamics. They found that at high densities a demixing transition takes place. However, at lower density values (or pressures), it is not possible to draw general conclusions regarding the phase behavior of these mixtures. This is due to a shortcoming stemming from the use of a fixed total density method with variable composition instead of the more natural (and appropriate) constant pressure approach.

Finally, Blair and Patey ${ }^{24}$ considered mixtures of neutral and DHSs and used different diameter ratios between the two components to study phase separation using Gibbs ensemble Monte Carlo ${ }^{25}$ simulation; they found that demixing takes place, at least, for high densities.

In this paper we will revisit the problem of the possible phase separation of HS/DHS mixtures. We will make use of efficient Monte Carlo methods to probe the existence of demixing and to locate the corresponding critical points resorting to finite-size scaling procedures. The influence of the pressure on the critical temperature of demixing will also be studied, and we will pay attention to the low pressure limit in order to further our knowledge on the LVE of pure DHS systems. In addition, we will assess the performance of two 
well known IETs, the hypernetted chain (HNC) and the RHNC approximations, when trying to cope with this relatively complex problem.

The models studied in this paper have some similarities with different spin fluid models ${ }^{26,27}$ and, to some extent, the appropriate theoretical and simulation tools to study both types of systems are similar. Nevertheless, there is a substantial physical difference between such spin fluids and the dipolar models studied here. Such a difference is the strong influence that the frustration ${ }^{28}$ that appears in the dipolar interactions causes on the phase diagram.

The rest of the paper is organized as follows. In Sec. II we will describe the systems under study and the thermodynamic relations that quantify and characterize the phase separation. In Sec. III we will discuss the computer simulation techniques used in the calculation. In Sec. IV we will discuss with some detail the analysis of the simulation results to compute phase equilibria and critical points. Section V is devoted to a brief account of the IETs. Finally, in Sec. VI we present and discuss our most significant results and outline the main conclusions of this work.

\section{THE SYSTEM}

We will consider mixtures of two species: neutral HSs (species 1) with dipolar moment $\mathcal{M}_{h}=0$ and DHSs (species 2) with dipolar moment $\mathcal{M}_{d} \equiv \mathcal{M}_{\text {DHS }} \neq 0$.

The potential energy is defined as a sum of pair interactions given by

$$
\begin{aligned}
u\left(\mathbf{r}_{i j}, \hat{\mathbf{S}}_{i}, \hat{\mathbf{S}}_{j}\right)= & u_{\mathrm{HS}}\left(r_{i j}\right)+\frac{\mathcal{M}_{i} \mathcal{M}_{j}}{4 \pi \epsilon_{0} r_{i j}^{[}}\left[\left(\hat{\mathbf{S}}_{i} \cdot \hat{\mathbf{S}}_{j}\right)-3\left(\hat{\mathbf{S}}_{i} \cdot \hat{\mathbf{r}}_{i j}\right)\right. \\
& \left.\times\left(\hat{\mathbf{S}}_{j} \cdot \hat{\mathbf{r}}_{i j}\right)\right]
\end{aligned}
$$

where $\hat{\mathbf{S}}_{k}$ and $\mathcal{M}_{k}$ are, respectively, the unit vector that define the dipole orientation and the magnitude of the dipolar moment of particle $k, \epsilon_{0}$ is the vacuum permittivity, $u_{\mathrm{HS}}(r)$ is the HS potential (for which we will here consider that the two species have the same diameter $\sigma$ ), and $\hat{\mathbf{r}}_{i j} \equiv \mathbf{r}_{i j} /\left|\mathbf{r}_{i j}\right|$, where $\mathbf{r}_{i j}$ is the vector joining the centers of particles $i$ and $j$.

Reduced units will be used throughout the paper, taking $\sigma$ as unit length and $u_{0}=\mathcal{M}_{d}^{2} /\left(4 \pi \epsilon_{0} \sigma^{3}\right)$ as the energy unit. The reduced temperature will be $T^{*}=k_{B} T / u_{0}$, with $k_{B}$ being Boltzmann's constant. We also define the reduced reciprocal temperature as $\beta^{*}=1 / T^{*}$. The reduced pressure will be given by $p^{*}=p \sigma^{3} / u_{0}$, but we will preferentially use as thermodynamic variable $(\beta p)^{*} \equiv\left(p \sigma^{3} / k_{B} T\right)=\left(p^{*} / T^{*}\right)$.

\section{A. Thermodynamics}

In order to explain the simulation methodologies used to compute the phase diagrams from a more convenient perspective, we will first recall the explicit form of the differential equation for the Gibbs energy function $G$ in a binary mixture,

$$
d(\beta G)=U d \beta+V d(\beta p)+\left(\beta \mu_{1}\right) d N_{1}+\left(\beta \mu_{2}\right) d N_{2},
$$

where $\beta=\left(k_{B} T\right)^{-1}, U$ is the internal energy, $V$ is the volume, and $\mu_{k}$ and $N_{k}$ are, respectively, the chemical potential and the number of particles of the species $k$. Taking into account that $N=N_{1}+N_{2}$, we can write

$$
d(\beta G)=U d \beta+V d(\beta p)+\left(\beta \mu_{1}\right) d N+(\beta \Delta \mu) d N_{2},
$$

where $\Delta \mu \equiv \mu_{2}-\mu_{1}$. A Legendre transformation leads to

$$
d(\beta \Upsilon)=U d \beta+V d(\beta p)+\left(\beta \mu_{1}\right) d N-N_{2} d(\beta \Delta \mu),
$$

where $Y=\mu_{1} N=G-N_{2} \Delta \mu$. According to Eq. (4), we can conveniently analyze the phase equilibria of the binary mixture in terms of four thermodynamic variables: $N, \beta, \beta p$, and $\beta \Delta \mu$. The only extensive variable, the total number of particles $N$, will define the system size.

According to Gibbs' phase rule, in conditions of thermodynamic equilibrium between two phases of two-component systems, only two out of the three intensive variables are independent. Let us assume that we fix temperature and pressure (or $\beta$ and $\beta p$ ). If under such conditions demixing takes place, locating the transition also implies computing the value of the remaining intensive variable at the transition, namely, $(\beta \Delta \mu)_{e}$.

On the other hand, assuming that phase separation can happen at low temperature, if we keep $(\beta p)$ constant and compute $(\beta \Delta \mu)_{e}$ as a function of $\beta$, we should expect the demixing transition to exhibit an upper critical temperature $T_{c}(\beta p)$ [or lower critical reciprocal temperature $\beta_{c}(\beta p)$ ], since in the limit $\beta \rightarrow 0$, we will reach a mixture of two species of HSs with equal diameters (i.e., a typical example of a fully miscible ideal mixture).

\section{SIMULATION TECHNIQUES}

In this work, we are dealing with dipolar interactions. This implies a considerable computational effort for the evaluation of interaction energies. Additionally, previous experience in the simulation of this type of systems ${ }^{3,8,13}$ indicates that the possible phase transitions should occur at relatively low temperatures. Both factors make advisable the use of the most efficient simulation procedures at hand in order to be able to draw meaningful conclusions from the calculations. Therefore, in what follows, we present in detail the special techniques that we have employed here to study the demixing transitions.

\section{A. Wang-Landau type algorithm}

We have devised a Wang-Landau ${ }^{29,30}$ type of algorithm tailored to our particular problem. This kind of procedures has already been successfully applied to the study of the fluid phase behavior of simple fluids ${ }^{31-34}$ and fluid mixtures. ${ }^{34} \mathrm{~A}$ number of advantages make this class of approaches a preferred tool for these problems. In particular, for the given conditions of $N, P$, and $T$, only one (long) simulation run is required instead of a number of different runs for different conditions of $x \equiv N_{2} / N$ (if one used the fixed composition $N P T$ ensemble) or $\Delta \beta \mu$ if the simulations are carried out in the semigrand NPT (SGNPT) ensemble. ${ }^{25}$ Moreover, the free energy barriers that make phase transitions difficult to handle in computer simulations are effectively removed by the na- 
ture of the method, and finally, it is quite simple to estimate how long a simulation run must be in order to reliably sample the system phase space.

Let us now recall the key features of the SGNPT algorithms so as to clarify how the Wang-Landau approach applies to our problem. A given configuration in a SGNPT ensemble has a probability given by

$$
\mathcal{P}(\mathcal{R}, \mathcal{S}, V) \propto V^{N} \exp \left[-\beta p V+\beta \Delta \mu N_{2}(\mathcal{S})-\beta \mathcal{U}(\mathcal{R}, \mathcal{S}, V)\right],
$$

where $\mathcal{R}$ denotes the system-size-reduced position coordinates of the particles, $\mathcal{S}$ represents the labels (species) of the $N$ particles, and $\mathcal{U}$ is the potential energy. The probability of finding a number $N_{2}$ of DHS can be extracted integrating Eq. (5) to get

$$
P\left(N_{2} \mid \beta \Delta \mu\right) \propto \exp \left[\beta \Delta \mu N_{2}\right] \exp \left[-\beta G\left(N, N_{2}, \beta p, \beta\right)\right],
$$

where $G\left(N, N_{2}, \beta p, \beta\right)$ is the Gibbs energy function, which can be decomposed into

$$
\beta G\left(N, N_{2}, \beta p, \beta\right)=\beta G_{0}\left(N, N_{2}, \beta p, \beta\right)+\beta G_{\mathrm{mix}}^{\mathrm{id}}\left(N_{2}, N\right),
$$

and the ideal Gibbs energy function of ideal mixture $\beta G_{\text {mix }}^{\mathrm{id}}$ is given by

$$
\begin{aligned}
\beta G_{\text {mix }}^{\mathrm{id}}\left(N_{2}, N\right) & =-\log \frac{N !}{\left.N_{2} !\left(N-N_{2}\right) !\right)} \\
& \simeq N[x \log x+(1-x) \log (1-x)] .
\end{aligned}
$$

Our aim here is to compute $\beta G_{0}$ as a function of $N_{2}$ for the given conditions of $N, \beta$, and $\beta p$. Notice than in an ideal mixture $\beta G_{0}$ does not depend on the composition $x$. In practical terms we will actually compute $\Delta \beta G_{0}$ defined as

$$
\begin{aligned}
\Delta \beta G_{0}\left(N_{2}, N, \beta p, \beta\right)= & \beta G_{0}\left(N_{2}, N, \beta p, \beta\right) \\
& -\beta G_{\mathrm{HS}}(N, \beta p, \beta),
\end{aligned}
$$

where $G_{\mathrm{HS}}$ stands for the Gibbs energy function of a pure HS system at the same conditions of temperature and pressure.

The Wang-Landau type simulations will be divided into two parts. The first part is the equilibration, which is devoted mainly to get a first estimation of $\Delta \beta G_{0}$ as a function of $N_{2}$. The second part will be referred as the sampling part, in which the actual averages are computed.

The simulation procedure involves three types of Monte Carlo moves: particle translations, volume changes, and changes in $N_{2}$. The orientational sampling will be coupled with the translation and identity change moves and will be presented later in the paper. Otherwise, the first two types of motions are performed using the standard procedures. ${ }^{25,35,36}$ The probability of a given configuration is

$$
\begin{aligned}
P_{\mathrm{WL}}(V, \mathcal{R}, \mathcal{S}) \propto & \frac{N_{2} !\left(N-N_{2}\right) !}{N !} V^{N} \exp \left[\beta \Omega_{0}\left(N_{2}\right)\right. \\
& -\beta p V-\beta \mathcal{U}],
\end{aligned}
$$

where $\mathcal{U}=\mathcal{U}(\mathcal{R}, \mathcal{S}, V)$ and $N_{2}=N_{2}(\mathcal{S})$. The weighting function $\Omega_{0}\left(N_{2}\right)$ is, in principle, an unknown function that is to be generated during the equilibration part of the procedure. The optimal choice for $\beta \Omega_{0}\left(N_{2}\right)$ is the one that leads to a flat distribution function $P_{\mathrm{WL}}\left(N_{2}\right)$. Integrating Eq. (10), one gets

$$
P_{\mathrm{WL}}\left(N_{2}\right) \propto \exp \left[\beta \Omega_{0}\left(N_{2}\right)\right] \exp \left[-\beta G_{0}\left(N_{2}\right)\right] .
$$

Therefore, our aim during the equilibration process will be the computation of a function $\beta \Omega_{0}\left(N_{2}\right)$ that fulfills

$$
\beta \Omega_{0}\left(N_{2}\right) \simeq \beta G_{0}\left(N_{2}\right)+c
$$

with $c$ being a constant. Hence, our procedure is to some extent similar to that used in SGNPT simulations ${ }^{25}$ except for two main details: first, the way in which different $N_{2}$ values are weighed [cf. Eqs. (10) and (5)], and second, during the equilibration part of the Wang-Landau procedure, the factor that weighs the different compositions is not constant, but it is being modified throughout the simulation so as to bias the sampling, and thus to get a flat distribution function $P_{\mathrm{WL}}\left(N_{2}\right)$. In this sense, the equilibration part is a nonequilibrium simulation, and one should be cautious not to draw quantitative conclusions at this initial level of the simulation.

\section{Wang-Landau equilibration section}

This part of the algorithm is split into several stages. Each stage is indexed by the subscript $s$. In the first stage an initial guess of $\beta \Omega_{0}\left(N_{2}\right)$ is required, for instance, $\beta \Omega_{0}\left(N_{2}\right)$ $=0$ for all $N_{2}$. The simulation is then run using the three types of moves described above. Once an identity change move is performed, the current value of the number of dipoles $N_{2}$ is used to update the stage histogram of the composition sampling $P_{s}\left(N_{2}\right)$. In addition the weighting function $\beta \Omega_{0}\left(N_{2}\right)$ is updated, such that

$$
\beta \Omega_{0}^{\text {new }}\left(N_{2}\right)=\beta \Omega_{0}^{\text {old }}\left(N_{2}\right)-\omega_{s},
$$

where $\omega_{s}$ depends on the equilibration stage via

$$
w_{s}=w_{1} \alpha^{s-1}, \quad \text { with } 0<\alpha<1 .
$$

The changes in $\beta \Omega_{0}\left(N_{2}\right)$ are intended to reduce the weight of the visited compositions, and thus ease the sampling of all the required mole fractions. An equilibration stage is finished when the corresponding $P_{s}\left(N_{2}\right)$ fulfills the convergence criteria given by

$$
\mathcal{P}_{s}\left(N_{2}\right) \equiv \frac{N_{2}^{\max }-N_{2}^{\min }+1}{\mathcal{N}_{t}} \mathcal{N}\left(N_{2}\right)>\delta
$$

for all $N_{2}$ in the sampling range of $N_{2}^{\min } \leq N_{2} \leq N_{2}^{\max }$ with $\delta$ being a prefixed convergence parameter that fulfills $(0<\delta$ $<1)$. In Eq. (15), $\mathcal{N}\left(N_{2}\right)$ is the number of occurrences of $N_{2}$ in the composition histogram over the whole stage, and $\mathcal{N}_{t}$ is the sum of $\mathcal{N}\left(N_{2}\right)$ over all the compositions in the sampling range. As the procedure advances into successive stages, the effect of updating $\beta \Omega_{0}\left(N_{2}\right)$ is less pronounced, so that in the final stages of the equilibration section, the simulation runs can be described as quasiequilibrium runs. Therefore, at the end of the equilibration part of the simulation, $\beta \Omega_{0}\left(N_{2}\right)$ is expected to be a good estimate of the Gibbs energy [in the sense of Eq. (12)]. We have typically used ten equilibration stages with $\alpha=0.25, \omega_{1}=0.262144, \omega_{10}=10^{-6}$, and $\delta=0.50$. 


\section{Sampling part}

Once the so-called equilibration part of the simulation is finished, we start the sampling of the system properties. Now, the weighting function $\beta \Omega_{0}\left(N_{2}\right)$ is kept fixed and, if the equilibration criterion has been adequately chosen, it is expected that the simulation runs will be able to sample the whole selected composition range.

This part of the simulation is split into blocks in order to facilitate the error analysis. With the results obtained during the sampling part, one can perform a further refinement of the Gibbs energy using

$$
\beta \Delta G_{0}\left(N_{2}\right)=\beta \Omega_{0}\left(N_{2}\right)-\log \left[\mathcal{P}\left(N_{2}\right)\right]+c,
$$

where $c$ is calculated by considering the constraint $\Delta \beta G_{0}\left(N_{2}=0\right)=0$.

\section{B. Computational details}

The simulations were carried out on cubic boxes with periodic boundary conditions (PBC). In order to take into account the long range nature of the dipolar interaction, we have used the Ewald sum technique ${ }^{25,35}$ using an inverse screening length $\kappa=5.75 / L$ and reciprocal vectors $2 \pi \mathbf{k} / L$ with $|\mathbf{k}| \leq k_{\max }=6$, where $L$ is the length of the simulation box.

After choosing representative values of $\beta p$, we first carried out some exploratory short simulation runs with small $N$ values to locate the corresponding critical temperatures. After these explorations we started long simulations for different values of $N$. The simulations were organized in cycles and, in each cycle, we perform $N$ one-particle translationalorientational moves, $N$ attempts of identity change, and one attempt of volume change.

The one-particle translation moves of DHS were coupled to a biased orientational sampling. This is carried out (provided that no overlap occurs in the translation attempt) by computing the electrical field that the rest of the particles of the system produces on both the current and the trial positions of the particle to be moved, say particle $i$. It is then possible $^{3,37}$ to integrate the orientational degrees of freedom of particle $i$ and compute the ratio between the probabilities of the two positions for the current configuration of the rest of the particles. These integrated probabilities are used to accept/reject the move. Then, using the electrical field at the chosen position, it is straightforward to select a new orientation of the dipole. ${ }^{38}$

A composition sampling step is carried out by first choosing at random (with equal probabilities) whether to increase or to decrease the number of DHS. If the selection is not directly forbidden by the prefixed range of compositions $0 \leq N_{2} \leq N_{2}^{\max }$, we pick at random one HS (if we are increasing $N_{2}$ ) or one DHS (if $N_{2}$ is to be reduced). Then the electrical field $\mathbf{E}$ at the position of the particle (excluding possible interactions with the periodic images of the chosen particle) is computed. Taking into account the value of $|\mathbf{E}|$, the interaction between the particle (for the DHS identity) with its periodical images (which depends on the volume, but not on the dipole orientation for a cubic box), and the values of $\beta \Omega_{0}$, it is straightforward ${ }^{3,37}$ to write down the acceptation criteria. As in the translational moves, if the new identity is that of a DHS, then a new orientation of the dipole is selected, taking into account the electrical field at the position of the particle.

\section{ANALYSIS OF THE SIMULATION RESULTS}

During the sampling part of the simulation, after each step of compositional sampling, we store the required information to build up different averages as functions of $N_{2}$. Essentially we accumulate results to compute averages as $\left\langle V^{k} U^{l}\right\rangle$ with $k=0, \cdots, 4$ and $l=0,2$, for each composition.

Utilizing these averages one can also estimate the thermodynamic properties in the vicinity of the simulated thermodynamic states by means of adequate Taylor expansions and the standard equations of the statistical thermodynamics. In practice we are interested in computing $\Delta \beta G\left(N_{2} \mid N, \beta p, \beta\right) \equiv \beta G\left(N_{2}, N, \beta p, \beta\right)-\beta G_{\mathrm{HS}}(N, \beta p)$. With the values of $\Delta \beta G\left(N_{2}\right)$ for constant $\beta, \beta p$, and $N$, we can analyze whether demixing occurs. For a given value of $\Delta \mu$ we can compute the corresponding distribution function,

$$
\mathcal{P}\left(N_{2} \mid \Delta \mu\right) \propto \exp \left[-\beta \Delta G\left(N_{2}\right)+\beta \Delta \mu N_{2}\right] .
$$

Considering that $x \equiv N_{2} / N$, the corresponding momenta $m_{k}$ $=m_{k}(\beta \Delta \mu)$ of the distributions $\mathcal{P}(x \mid \Delta \mu)$ can be evaluated as

$$
\begin{aligned}
& m_{1}=\bar{x}=\langle x\rangle, \\
& m_{k}=\left\langle(x-\bar{x})^{k}\right\rangle \quad(k=2,3,4) .
\end{aligned}
$$

In order to check for the presence of possible first order demixing transitions, we determine $\beta \Delta \mu_{e}(\beta, \beta p, N)$ as the value of $\beta \Delta \mu$ that maximizes $m_{2}$ at the corresponding conditions $(\beta, \beta p, N)$. Then, it is convenient to analyze the system size dependence of the distributions at $\Delta \mu_{e}$, with fixed conditions of temperature and pressure, so as to extrapolate the system behavior at the thermodynamic limit. From the distributions at $\Delta \mu_{e}(\beta, \beta p, N)$, we compute the following quantities:

$$
\begin{aligned}
x_{m}(N) & =m_{1}\left(N, \beta \Delta \mu_{e}\right), \\
\delta x(N) & =\left[m_{2}\left(N, \beta \Delta \mu_{e}\right)\right]^{1 / 2}, \\
G_{4}(N) & =\frac{1}{2}\left[3-\frac{m_{4}\left(N, \beta \Delta \mu_{e}\right)}{\left[m_{2}\left(N, \beta \Delta \mu_{e}\right)\right]^{2}}\right],
\end{aligned}
$$

and analyze their dependence on the system size, represented either with $N$ or the related quantity $\mathcal{L} \equiv N^{1 / 3}$. Note that the condition of maximum for $m_{2}$ implies that the third moment of the composition distribution fulfills $m_{3}\left(N, \beta \Delta \mu_{e}\right)=0$. The extrapolation of the results to $N \rightarrow \infty$ will define our estimations at the thermodynamic limit, [e.g., $\left.x_{m}=\lim _{N \rightarrow \infty} x_{m}(N)\right]$. The mole fraction fluctuation $\delta x(N)$ is expected to behave as

$$
\begin{aligned}
& \lim _{N \rightarrow \infty} \delta x(N)= \begin{cases}\delta x>0, & \beta>\beta_{c}(\beta p) \\
\delta x=0, & \beta \leq \beta_{c}(\beta p),\end{cases} \\
& \delta x(N) \propto N^{-1 / 2}=\mathcal{L}^{-3 / 2}, \quad \beta<\beta_{c}(\beta p),
\end{aligned}
$$




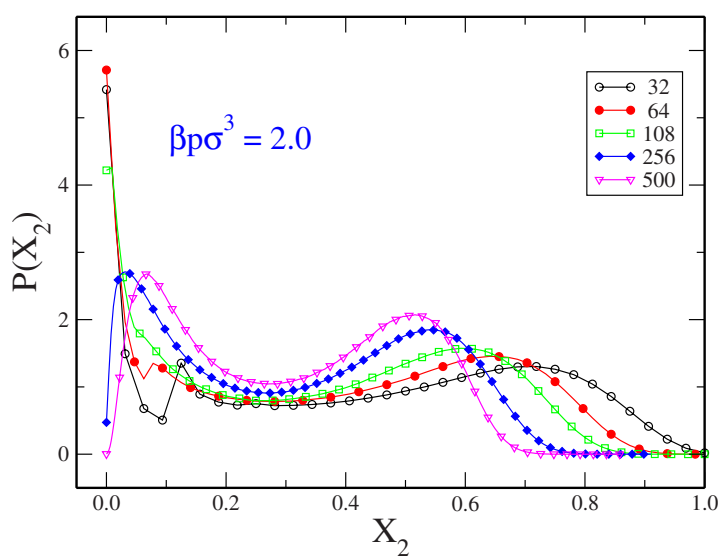

FIG. 1. (Color online) Composition distribution function at criticality with $(\beta p)^{*}=2.0$. For each system size the distribution is plotted at its pseudocritical conditions $\beta_{c}(N)$ and $\Delta \mu_{c}(N)$. See text for details.

$$
\delta x(N)-\delta x \sim N^{-1}=\mathcal{L}^{-3}, \quad \beta>\beta_{c}(\beta p),
$$

whereas exactly at the critical point the expected scaling behavior is ${ }^{36}$

$$
\delta x(\mathcal{L}) \propto \mathcal{L}^{-\beta^{\prime} / \nu}, \quad \beta=\beta_{c}(\beta p),
$$

where $\beta^{\prime}$ and $\nu$ are critical exponents. The prime in the exponent $\beta^{\prime}$ is introduced to distinguish it from the inverse temperature $\beta$. The values of these critical exponents have been taken to be ${ }^{39,40} \beta^{\prime}=0.326$ and $\nu=0.630$.

For $\beta>\beta_{c}(\beta p)$ the composition of the two phases at equilibrium will be given by

$$
x_{\alpha}=x_{m} \pm \delta x,
$$

where $x_{m}$ and $\delta x$ are extrapolated by fitting the results for $x_{m}(N)$ and $\delta x(N)$ to polynomials of $(1 / N)$ up to the first or second order in $(1 / N)$.

The quantity $G_{4}(N)$, defined through the second and fourth moments of the mole fraction probability distribution at $\left(\beta \Delta \mu_{e}\right)$, is closely related to the reduced fourth order cumulant of the distribution. ${ }^{36,41,42}$ The behavior of $G_{4}(N)$ with the system size is expected to fulfill ${ }^{36,42}$

$$
\lim _{N \rightarrow \infty} G_{4}(N)=\left\{\begin{array}{cc}
1, & \beta>\beta_{c}(\beta p) \\
G_{c}, & \beta=\beta_{c}(\beta p) \\
0, & \beta \leq \beta_{c}(\beta p),
\end{array}\right.
$$

where $G_{c}$ is a singular value, which depends on the geometry, symmetry, and boundary conditions of the finite size systems and on the class of universality of the critical behavior of the system. ${ }^{42,43}$ For systems with critical behavior belonging to the three dimensional (3D) Ising universality class that are simulated on cubic boxes with $\mathrm{PBC}$, we have ${ }^{43} G_{c}$ $\approx 0.698$.

According to the usual finite-size scaling techniques, one expects that for large systems $G_{4}(N)$ becomes invariant with the system size and equal to $G_{c}$ at the critical line. Taking this into account, one can define pseudocritical inverse temperatures $\beta_{c}(N)$ for finite system sizes as

$$
G_{4}\left(\beta_{c}(N), N \mid \beta p\right)=G_{c} .
$$

In Figs. 1 and 2 we present the mole fraction probability

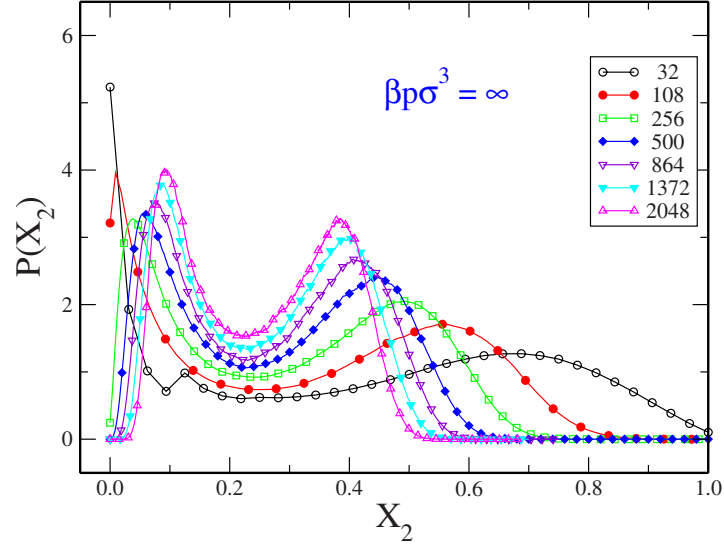

FIG. 2. (Color online) Composition distribution function at criticality in the infinite pressure limit (fcc lattice). For each system size the distribution is plotted at its pseudocritical conditions $\beta_{c}(N)$ and $\Delta \mu_{c}(N)$. See text for details.

distributions $\mathcal{P}\left(x \mid \Delta \mu_{e}\right)$ at the pseudocritical temperatures for different system sizes and two values of the reduced pressure $\beta p$. It can be seen that as the system size increases, the shape of these distributions approaches the universal curve. ${ }^{42}$

According to finite-size scaling procedures, ${ }^{42,44}$ the following asymptotic behavior is expected for $\beta_{c}(N)$ :

$$
\beta_{c}(\mathcal{L})=\beta_{c}+a_{\beta} \mathcal{L}^{-(1+\theta) / \nu},
$$

where we have used the length $\mathcal{L}$ as a measure of the system size; $\theta$ is a critical exponent, which for the Ising-3D universality class is estimated to be ${ }^{40} \theta \simeq 0.52$. Once one has $\beta_{c}(\mathcal{L})$ and the corresponding $\beta \Delta \mu_{c}(\mathcal{L})$, using the appropriate reweighting strategies one can compute at those conditions the system size dependent pseudocritical quantities $\rho_{c}(\mathcal{L}), x_{c}(\mathcal{L})$, and $\delta x_{c}(\mathcal{L})$. These quantities are expected to fulfill for not too small systems ${ }^{42,44}$

$$
\begin{aligned}
& x_{c}(\mathcal{L}) \simeq x_{c}+a_{x} \mathcal{L}^{-(d-1 / \nu)}, \\
& \rho_{c}(\mathcal{L}) \simeq \rho_{c}+a_{\rho} \mathcal{L}^{-(d-1 / \nu)}, \\
& \delta x_{c}(\mathcal{L}) \simeq a_{\delta} \mathcal{L}^{-\beta^{\prime} / \nu},
\end{aligned}
$$

where $d$ is the dimensionality of the system.

In the mixtures we are dealing with, the two phases at equilibrium have different total densities. In fact, for a given pressure and temperature, the total density composition is strongly correlated; the total density increases in parallel with the mole fraction of the DHSs. Bearing this in mind, we can estimate the total density of the phases at equilibrium using the same procedure employed for the compositions.

\section{INTEGRAL EQUATION THEORIES}

In what follows we will briefly sketch the key equations that build up both the RHNC and HNC approximations. Both approaches start from the Ornstein-Zernike equation that can be expressed for a mixture of molecular fluids as 


$$
h_{\alpha \beta}(12)=c_{\alpha \beta}(12)+\sum_{\lambda} \frac{\rho_{\lambda}}{\Omega} \int c_{\alpha \lambda}(13) h_{\lambda \beta}(32) d \omega_{3} d \mathbf{r}_{3},
$$

where different species are denoted by Greek indices, $\omega_{i}$ represents the orientation of particle $i, \mathbf{r}_{\mathbf{i}}$ is the vector denoting the position of the particle, and $\Omega$ is $4 \pi$ for linear molecules and $8 \pi^{2}$ in the nonlinear case. The functions $h(i j)$ and $c(i j)$ are the total and direct correlation functions and $\rho_{\lambda}$ is the number density of the species $\lambda$. Equation (34) must be supplemented by a closure, which in its general form reads

$$
\begin{aligned}
h_{\alpha \beta}(12)= & \exp \left[-\beta u_{\alpha \beta}(12)+h_{\alpha \beta}(12)\right. \\
& \left.-c_{\alpha \beta}(12)+b_{\alpha \beta}(12)\right]-1,
\end{aligned}
$$

where $b_{\alpha \beta}(12)$ is the so-called bridge function. Here we will only consider two approximations, the simplest HNC, which implies $b_{\alpha \beta}(12)=0$ and the RHNC in which the bridge function of the system under consideration is approximated by that of a reference system. Since we are dealing with equisized HSs (plus an extra multipole contribution whose angular average contribution vanishes), the most appropriate choice of the reference system is the HS fluid of diameter $\sigma$ and density $\rho=\Sigma_{\lambda} \rho_{\lambda}$. Now, one can proceed to solve Eqs. (34) and (35) using the standard spherical harmonic expansion of the correlation functions. ${ }^{45}$ Thus one would obtain a solution for a given composition, total density, and temperature. However, for the purpose of studying phase separation in mixtures, it is certainly better to work at constant pressure. In our case, the pressure is straightforward to calculate through the virial theorem, namely,

$$
\begin{aligned}
\beta p= & \sum_{\lambda} \rho x_{\lambda} \\
& -\frac{2 \pi \rho^{2}}{3} \beta \sum_{\alpha \lambda} x_{\lambda} x_{\alpha} \sum_{l_{1} l_{2} l_{m}} \int_{0}^{\infty} d r r^{3} \frac{d u_{l_{1} l_{2} m}^{\alpha \lambda}(r)}{d r} g_{l_{1} l_{2} m}^{\alpha \lambda}(r),
\end{aligned}
$$

where $f_{l_{1} l_{2} m}^{\alpha \lambda}(r)$ are the coefficients of the spherical harmonic expansion of the angular functions correlating particles of species $\alpha$ and $\lambda$ in the molecular reference frame. The way to proceed is then to solve Eqs. (34) and (35) coupled with Eq. (36) for the given $\beta, \beta p$, and composition, for both the correlation functions $h$ and $c$, and the total density $\rho$ following the ideas of Pastore et al. ${ }^{46}$ This can be easily accomplished if the nonlinear system of equations is solved using Fries and Cosnard's version of the general minimization of residues (GMRES) algorithm. ${ }^{47}$ We have checked the results of our algorithm against constant density calculations and with the results of Chen et al. ${ }^{20}$ and Range and Klapp. ${ }^{21}$ In all cases the agreement found was satisfactory and the stability limits found in Refs. 20 and 21 were reproduced.

Once the equations are solved at constant pressure, one can also evaluate thermodynamic quantities such as internal energies, Helmholtz free energies, and chemical potentials. ${ }^{45,48}$ In this way, it should be possible, in principle, to determine the conditions of phase separation solving for equal pressure and chemical potentials. We have found, how-
TABLE I. Phase coexistence at $(\beta p)^{*}=5.0, x$ is the mole fraction of DHSs, and the indices I and II refer to the two phases. Error bars are given between parentheses in units of the last figure of the property and correspond to a confidence level of about $95 \%$.

\begin{tabular}{lllll}
\hline \hline \multicolumn{1}{c}{$\beta^{*}$} & \multicolumn{1}{c}{$x^{\mathrm{I}}$} & \multicolumn{1}{c}{$x^{\mathrm{II}}$} & $N \sigma^{3} /\langle V\rangle_{\mathrm{I}}$ & $N \sigma^{3} /\langle V\rangle_{\mathrm{II}}$ \\
\hline 4.2 & $0.01(1)$ & $0.74(1)$ & $0.752(4)$ & $0.831(4)$ \\
4.0 & $0.03(2)$ & $0.66(2)$ & $0.751(6)$ & $0.817(7)$ \\
3.8 & $0.07(1)$ & $0.57(1)$ & $0.754(4)$ & $0.803(4)$ \\
$3.61(4)$ & $0.263(13)$ & $0.263(13)$ & $0.768(3)$ & $0.768(3)$ \\
\hline \hline
\end{tabular}

ever, that the integral equation breaks down at low temperatures-low dipole concentrations before the binodal curve is reached. This shortcoming is most likely connected with the inability of HNC-like integral equations to furnish convergent solutions in the strong association regime. ${ }^{49,50}$ Thus, in this work we have restricted ourselves to probe to boundaries of the no-solution region, and so we will provide some rough estimates of the location of the critical point based on the position of the maxima of the corresponding no-solution curves. These estimates will of course overestimate the simulated critical temperatures. ${ }^{51}$ Here it should be stressed that the free energy and virial pressure are fully consistent ${ }^{52}$ in the HNC approximation. Incorporation of a HS bridge function in the RHNC hardly worsens this consistency, but considerably improves the consistency between virial pressure and fluctuation theorem isotherm compressibility. However, at high densities/pressures for which demixing is to be expected, there remains a certain amount of inconsistency (up to a 20\%) that can compromise the accuracy of the calculation to certain extent. In any case, the aforementioned convergence problems of the integral equation represent a much more severe drawback.

\section{RESULTS}

We have carried out simulations to compute phase transitions and critical points of the fluid-fluid equilibria at different values of $\beta p$. In addition we have performed a similar calculation for a face centered cubic (fcc) close packing distribution of spheres, which in practice can be viewed as a lattice gas model with dipolar interactions. In Table I we present the simulation results for the fluid-fluid equilibrium at $(\beta p)^{*}=5.0$. These results were computed by performing simulations for several system sizes $N=32,64,108,256$, and 500 and extrapolating to the thermodynamic limit. In addition we include the estimation of the critical properties at the same conditions. It is clear from these results that the coexistence curve is very asymmetric in both $x_{2}-T$ and $\rho-T$ planes. One of the phases, phase I, is a much diluted solution of DHS in a HS system, whereas in phase II the mole fraction of HS is relatively large. This fact is also reflected in the values of the critical properties $x_{c}<1 / 2$ and the critical density $\rho_{c}$ is close to the value of a pure HS system at the same pressure.

The search for critical temperatures was carried as follows. Once $\beta p$ is fixed, we paid attention to the behavior of relatively small systems $N=32$. By means of several short simulations and the histogram analysis, we could get the ap- 
TABLE II. Pseudocritical points of the fluid mixtures. Error bars are given as in Table I.

\begin{tabular}{|c|c|c|c|c|c|c|}
\hline$(\beta p)^{*}$ & $N$ & $\beta_{c}^{*}(N)$ & $x_{c}(N)$ & $\rho_{c}(N) \sigma^{3}$ & $\rho_{2 c}(N) \sigma^{3}$ & $\delta x_{c}(N) \mathcal{L}^{\beta^{\prime} / \nu}$ \\
\hline \multirow[t]{5}{*}{5.0} & 32 & $3.701(18)$ & $0.3973(23)$ & $0.7854(14)$ & $0.3210(24)$ & $0.535(3)$ \\
\hline & 64 & $3.693(13)$ & $0.3615(15)$ & $0.7804(11)$ & $0.2885(14)$ & $0.533(2)$ \\
\hline & 108 & $3.673(11)$ & $0.3421(23)$ & $0.7782(13)$ & $0.2714(21)$ & $0.537(4)$ \\
\hline & 256 & $3.645(23)$ & $0.313(3)$ & $0.7748(14)$ & $0.2460(28)$ & $0.534(6)$ \\
\hline & 500 & $3.623(14)$ & $0.301(7)$ & $0.7729(13)$ & $0.235(8)$ & $0.54(2)$ \\
\hline \multirow[t]{5}{*}{4.0} & 32 & $3.841(20)$ & $0.3949(21)$ & $0.7365(12)$ & $0.3003(22)$ & $0.534(3)$ \\
\hline & 64 & $3.813(15)$ & $0.3655(16)$ & $0.7333(11)$ & $0.2754(15)$ & $0.539(3)$ \\
\hline & 108 & $3.792(10)$ & $0.346(3)$ & $0.7317(9)$ & $0.2592(27)$ & $0.542(4)$ \\
\hline & 256 & $3.745(15)$ & $0.318(4)$ & $0.7276(9)$ & $0.236(4)$ & $0.539(8)$ \\
\hline & 500 & $3.74(4)$ & $0.306(12)$ & $0.7264(23)$ & $0.226(9)$ & $0.54(3)$ \\
\hline \multirow[t]{5}{*}{2.0} & 32 & $4.30(3)$ & $0.406(5)$ & $0.5978(21)$ & $0.256(4)$ & $0.545(5)$ \\
\hline & 64 & $4.20(3)$ & $0.376(4)$ & $0.5926(12)$ & $0.2335(27)$ & $0.552(4)$ \\
\hline & 108 & $4.17(5)$ & $0.356(6)$ & $0.5894(18)$ & $0.219(4)$ & $0.56(1)$ \\
\hline & 256 & $4.138(22)$ & $0.329(6)$ & $0.5840(15)$ & $0.199(5)$ & $0.56(1)$ \\
\hline & 500 & $4.10(3)$ & $0.318(13)$ & $0.5818(29)$ & $0.190(9)$ & $0.56(2)$ \\
\hline \multirow[t]{5}{*}{1.0} & 32 & $4.52(5)$ & $0.408(3)$ & $0.4690(15)$ & $0.2088(19)$ & $0.548(5)$ \\
\hline & 64 & 4.60(7) & $0.390(6)$ & $0.463(4)$ & $0.195(5)$ & $0.572(7)$ \\
\hline & 108 & 4.62(9) & $0.383(13)$ & $0.462(9)$ & $0.191(8)$ & $0.60(2)$ \\
\hline & 256 & $4.56(6)$ & $0.345(12)$ & $0.4512(19)$ & $0.165(7)$ & $0.59(3)$ \\
\hline & 500 & $4.57(13)$ & $0.33(4)$ & $0.446(10)$ & $0.153(19)$ & $0.60(6)$ \\
\hline \multirow[t]{4}{*}{0.5} & 32 & $5.03(10)$ & $0.437(7)$ & $0.3608(27)$ & $0.182(4)$ & $0.577(8)$ \\
\hline & 64 & $5.08(8)$ & $0.401(6)$ & $0.3477(24)$ & $0.158(4)$ & $0.59(1)$ \\
\hline & 108 & $5.13(11)$ & $0.382(20)$ & $0.340(6)$ & $0.145(10)$ & $0.61(3)$ \\
\hline & 256 & $5.05(11)$ & $0.353(20)$ & $0.330(5)$ & $0.127(14)$ & $0.60(4)$ \\
\hline
\end{tabular}

proximate location of the critical region. This could be done due to the fact that in spite of the small sample size, the results for the pseudocritical values $\beta_{c}(N, \beta p)$ for $N=32$ are usually very close (say, less than $5 \%$ apart) to the extrapolated value at the thermodynamic limit (see Tables II-IV).

After having a good guess about the pseudocritical conditions, we run long simulations and performed the histogram analysis to compute more precisely the pseudocritical conditions for the smallest systems. Then, we subsequently run simulations with larger number of particles, usually $N$ $=64,108,256$, and 500, taking advantage in each case of the information from previous (smaller) sizes, so as to choose the simulation temperature, and eventually to reduce, when possible, the maximum value of $N_{2}$ to be sampled in the simulation runs.

Despite the use of the special procedures devised to improve the orientational sampling, long runs were required to extract reliable information regarding the demixing transition. One of the main problems found in the simulation is the strong density-composition correlation (at constant $T$ and $p$ ) that appears in these fluid mixtures. This implies that a large range of densities has to be sampled at constant pressure conditions. In these circumstances, the hard core of the model induces a slow sampling of the density fluctuations. This fact, together with the high computational cost due to the presence of multipoles, made unfeasible the simulation of very large systems. As an example, a precise estimation of the pseudocritical temperature for $N=500$ at the lower value of $\beta p$ using a reasonable amount of CPU time was already too hard to obtain. However, in the particular case of the lattice system studied as a reference, the low value of $\beta_{c}$ and above all the fact that the calculations are performed at constant volume made possible the simulation of much larger systems.

In Tables II-IV we show the results for the pseudocritical and extrapolated critical behaviors of the different systems. It can be noticed that the results for the fluid phases are nearly consistent with the scaling relation given in Eq. (33). This is somewhat surprising given the relative small size of the systems considered.

TABLE III. Pseudocritical points of the fcc lattice system. Error bars are given as in Table I.

\begin{tabular}{llllllll}
\hline \hline$N$ & \multicolumn{1}{c}{32} & \multicolumn{1}{c}{108} & \multicolumn{1}{c}{256} & \multicolumn{1}{c}{500} & \multicolumn{1}{c}{864} & \multicolumn{1}{c}{1372} & \multicolumn{1}{c}{2048} \\
\hline$\beta_{c}^{*}(N)$ & $2.476(4)$ & $2.444(6)$ & $2.429(3)$ & $2.417(3)$ & $2.414(4)$ & $2.409(3)$ & $2.405(4)$ \\
$x_{c}(N)$ & $0.4121(10)$ & $0.3418(19)$ & $0.3052(14)$ & $0.2814(16)$ & $0.2691(20)$ & $0.2602(14)$ & $0.2538(20)$ \\
$\delta x_{c}(N) \mathcal{L}^{\beta^{\prime} / \nu}$ & $0.560(2)$ & $0.539(4)$ & $0.519(3)$ & $0.498(3)$ & $0.490(2)$ & $0.484(2)$ & $0.478(7)$ \\
\hline \hline
\end{tabular}


TABLE IV. Estimations of the critical points. Error bars are given as in Table I.

\begin{tabular}{lllll}
\hline \hline$(\beta p)^{*}$ & \multicolumn{1}{c}{$\beta_{c}$} & \multicolumn{1}{c}{$x_{c}$} & \multicolumn{1}{c}{$\rho_{c} \sigma^{3}$} & \multicolumn{1}{c}{$\rho_{2 c} \sigma^{3}$} \\
\hline$\infty(\mathrm{fcc})$ & $2.403(4)$ & $0.223(4)$ & \multicolumn{1}{c}{$\sqrt{2}$} & $0.315(6)$ \\
5.0 & $3.61(4)$ & $0.263(13)$ & $0.768(3)$ & $0.203(6)$ \\
4.0 & $3.72(4)$ & $0.269(17)$ & $0.722(4)$ & $0.195(14)$ \\
2.0 & $4.09(4)$ & $0.288(13)$ & $0.576(4)$ & $0.167(10)$ \\
1.0 & $4.60(10)$ & $0.30(5)$ & $0.431(5)$ & $0.13(3)$ \\
0.5 & $5.07(7)$ & $0.31(5)$ & $0.313(15)$ & $0.10(3)$ \\
\hline \hline
\end{tabular}

In Figs. 1 and 2 it can be seen that for small system sizes $N=32$ and $N=64$ a spurious peak appears at low values of $x_{2}$ in the composition probability distributions at pseudocritical conditions. This effect is more important for low pressures (which also imply low critical temperatures) and it is likely due to the energy stabilization provided for the percolation through the simulation box of linear chains of dipoles. Such a percolation effect is thought to be responsible for the difficulties encountered when simulating dipolar systems at low densities and low temperatures. ${ }^{13}$

When comparing the results shown in Table I for the coexisting compositions at various temperatures for $\beta p \sigma^{3}$ $=5.0$ with the no-solution curves of the HNC/RHNC approximations depicted in Fig. 3, it is obvious that the theory overestimates the critical temperature even if the asymmetry of the phase separation is somehow captured.

Paying attention to Table IV, we can observe that the increase in $(\beta p)$ (or the pressure) leads to an increase in the critical temperature (i.e., reduction in $\beta_{c}$ ), together with a smaller value for the DHS mole fraction at the critical point $x_{c}$. Focusing on the values at $\beta p \sigma^{3}=2.0$, we observe that the estimate $\beta_{c}^{*}=4.09 \pm 0.04$ agrees well with the result previously reported by Blair and Patey ${ }^{24}\left(\beta_{c}^{*} \simeq 4.0 \pm 0.2\right)$. The pressure dependence of the critical parameters is found to be in qualitative agreement with the pseudocritical values obtained from the RHNC and HNC integral equations [see Fig. 3]. A quantitative comparison can be seen in Fig. 4, where

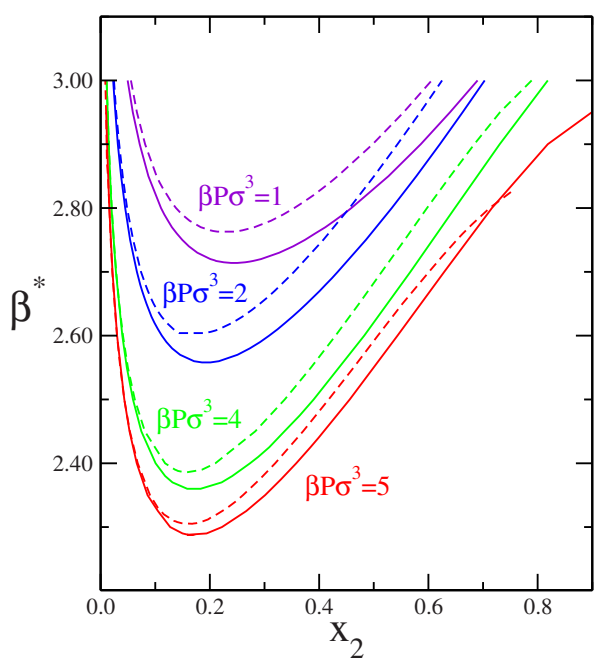

FIG. 3. (Color online) No-solution curves of the HNC/RHNC approximations for various pressures. The solid and dashed curves correspond to the HNC and RHNC approximations, respectively.

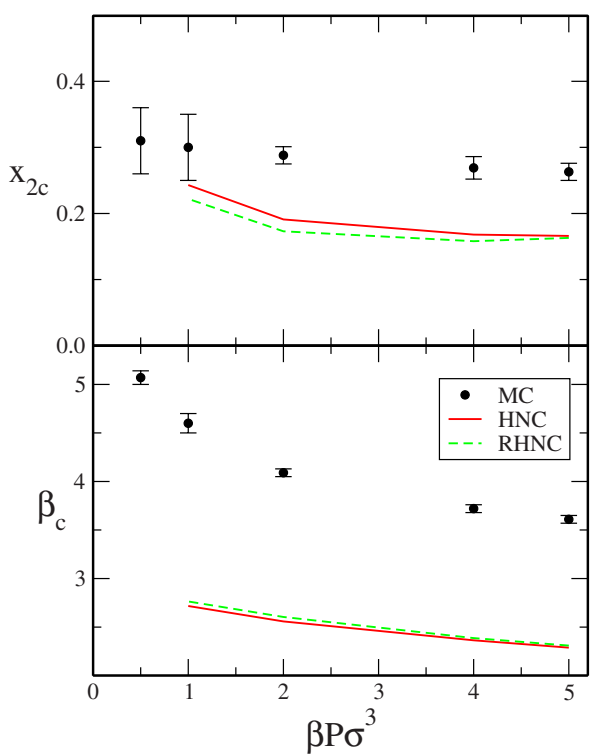

FIG. 4. (Color online) Pressure dependence of the inverse critical temperature $\beta_{c}$ and mole fraction $x_{2}$ from the simulation (symbols) and in the HNC/RHNC approximation (curves). Error bars correspond to a confidence level of $95 \%$.

we observe that, in particular, the $\beta_{c}$ are rather underestimated (as mentioned above). As the pressure is lowered the deviations increase. This is most likely due to the fact that one encounters the well known limitations of the $\mathrm{HNC/}$ RHNC for systems in which association and/or chaining are present at low temperatures, such as dipolar and ionic fluids.

In consonance with the discussion above, we also observed that as we decrease the reduced pressure, it becomes more difficult for the simulation procedure to find the conditions of demixing. In practice we are, somehow, moving from a typical demixing transition into a liquid-vapor-like equilibrium since the $x_{c}$ increases as the pressure decreases. The same effect was found by Ganzenmüller and Camp ${ }^{13}$ and by Blair and Patey ${ }^{24}$ when approaching the pure DHS limit from different routes.

Following the ideas of Ganzenmüller and $\mathrm{Camp}^{13}$ we

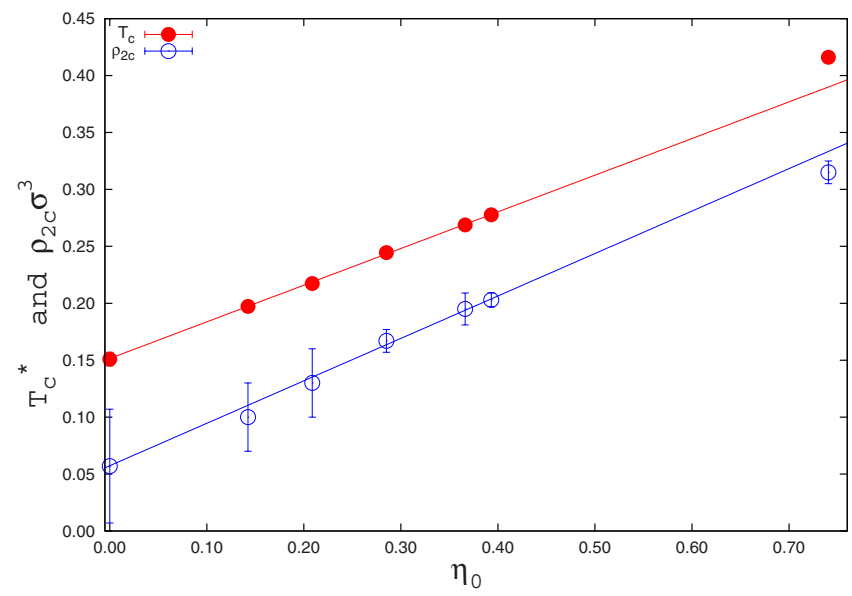

FIG. 5. (Color online) Reduced critical temperatures and densities as a function of the isobaric density of HSs (see text for details). Lines correspond to least squares fittings of the simulation results for fluid phases 0.1 $<\eta_{0}<0.5$. The points for $\eta_{0}=0$ are the extrapolation values from that fitting. Error bars correspond to a confidence level of $95 \%$. 
can analyze the trends of the critical temperature and the critical density of DHS $\rho_{2 c}$ as the concentration of the apolar component decreases. In Fig. 5 we plot both quantities $T_{c}^{*}$ and $\rho_{c 2}^{*}$ as a function of the packing fraction $\eta_{0}$ of a pure HS fluid at the corresponding value of $(\beta p)^{*}$. In the limit $\eta_{0}$ $\rightarrow 0$, we recover the pure DHS system. A rough analysis of the possible criticality of the pure DHS system can be performed by extrapolation of the corresponding critical property assuming a linear dependence with $\eta_{1}$. Surprisingly, the extrapolated value for the critical temperature we obtain, $T_{c}^{*}\left(\eta_{0}=0\right) \simeq 0.153 \pm 0.003$, agrees with the value reported by Ganzenmüller and $\mathrm{Camp}^{13}$ 0.151(1); whereas the result for the limiting value of $\rho_{c 2}^{*}\left(\eta_{0}\right), 0.06 \pm 0.05$ is not far from the value $\rho_{c 2}^{*} \simeq 0.1$ reported in the same paper.

Regarding the existence of LVE in pure DHS system, in spite of the extrapolations performed above, we cannot give a clear answer; the conditions of the mixtures studied in this paper are, even for the lower values of pressure, still far from pure system conditions. A full calculation of the critical conditions for lower pressures, including finite-size scaling, becomes too demanding from the computational standpoint.

It is also possible that aside from the typical LVE or the transition between linear chains and branching ${ }^{5}$ explanations for the low temperature phase behavior of pure DHS, a third scenario has to be taken into account. The results of Hynninen and Dijkstra ${ }^{15,16}$ seem to suggest that a possible LVE could be pre-empted by a gas-solid transition. Nevertheless, the investigation of such a possibility is clearly beyond the scope of the present work.

\section{ACKNOWLEDGMENTS}

The authors gratefully acknowledge the support from the Dirección General de Investigación Científica y Técnica under Grant No. MAT2007-65711-C04-04 and from the Dirección General de Universidades e Investigación de la Comunidad de Madrid under Grant No. S0505/ESP/0299 and Program MOSSNOHO-CM.

${ }^{1}$ D. Wei and G. N. Patey, Phys. Rev. Lett. 68, 2043 (1992).

${ }^{2}$ D. Wei and G. N. Patey, Phys. Rev. A 46, 7783 (1992).

${ }^{3}$ J. M. Caillol, J. Chem. Phys. 98, 9835 (1993).

${ }^{4}$ M. E. van Leeuwen and B. Smit, Phys. Rev. Lett. 71, 3991 (1993).

${ }^{5}$ J. J. Weis and D. Levesque, Phys. Rev. Lett. 71, 2729 (1993).

${ }^{6}$ D. Levesque and J. J. Weis, Phys. Rev. E 49, 5131 (1994).

${ }^{7}$ J. C. Shelley, G. N. Patey, D. Levesque, and J. J. Weis, Phys. Rev. E 59, 3065 (1999)

${ }^{8}$ P. J. Camp and G. N. Patey, Phys. Rev. E 62, 5403 (2000).

${ }^{9}$ P. J. Camp, J. C. Shelley, and G. N. Patey, Phys. Rev. Lett. 84, 115 (2000).

${ }^{10}$ J. M. Tavares, M. M. Telo da Gama, and M. A. Osipov, Phys. Rev. E 56, R6252 (1997).

${ }^{11}$ S. Klapp and F. Forstmann, J. Chem. Phys. 106, 9742 (1997).

${ }^{12}$ C. Holm and J.-J. Weis, Curr. Opin. Colloid Interface Sci. 10, 133 (2005).
${ }^{13}$ G. Ganzenmüller and P. J. Camp, J. Chem. Phys. 126, 191104 (2007).

${ }^{14}$ A. F. Pshenichnikov and V. V. Mekhonoshin, Eur. Phys. J. E 6, 399 (2001).

${ }^{15}$ A.-P. Hynninen and M. Dijkstra, Phys. Rev. Lett. 94, 138303 (2005).

${ }^{16}$ A.-P. Hynninen and M. Dijkstra, Phys. Rev. E 72, 051402 (2005).

${ }^{17}$ R. Hentschke, J. Bartke, and F. Pesth, Phys. Rev. E 75, 011506 (2007).

${ }^{18}$ A. O. Ivanov, S. S. Kantorovich, and P. J. Camp, Phys. Rev. E 77, 013501 (2008).

${ }^{19}$ R. Hentschke and J. Bartke, Phys. Rev. E 77, 013502 (2008).

${ }^{20}$ X. S. Chen, M. Kasch, and F. Forstmann, Phys. Rev. Lett. 67, 2674 (1991).

${ }^{21}$ G. M. Range and S. H. L. Klapp, Phys. Rev. E 69, 041201 (2004).

${ }^{22}$ I. Szalai and S. Dietrich, Mol. Phys. 103, 2873 (2005).

${ }^{23}$ S. W. de Leeuw, B. Smit, and C. P. Williams, J. Chem. Phys. 93, 2704 (1990).

${ }^{24}$ M. J. Blair and G. N. Patey, Phys. Rev. E 57, 5682 (1998).

${ }^{25}$ D. Frenkel and B. Smit, Understanding Molecular Simulation, 2nd ed. (Academic, London, 2002).

${ }^{26}$ I. P. Omelyan, I. M. Mryglod, R. Folk, and W. Fenz, Phys. Rev. E 69, 061506 (2004); I. P. Omelyan, I. M. Mryglod, R. Folk, and W. Fenz, Phys. Rev. Lett. 94, 045701 (2005); W. Fenz, R. Folk, I. M. Mryglod, and I. P. Omelyan, Phys. Rev. E 68, 061510 (2003).

${ }^{27}$ E. Lomba, C. Martín, N. G. Almarza, and F. Lado, Phys. Rev. E 74, 021503 (2006).

${ }^{28}$ Frustrated Spin Systems, edited by H. T. Diep (World Scientific, Singapore, 2004).

${ }^{29}$ F. Wang and D. P. Landau, Phys. Rev. Lett. 86, 2050 (2001).

${ }^{30}$ F. Wang and D. P. Landau, Phys. Rev. E 64, 056101 (2001).

${ }^{31}$ E. Lomba, C. Martín, N. G. Almarza, and F. Lado, Phys. Rev. E 71, 046132 (2005).

${ }^{32}$ E. Lomba, N. G. Almarza, C. Martín, and C. McBride, J. Chem. Phys. 126, 244510 (2007).

${ }^{33}$ E. Lomba, N. G. Almarza, and C. Martín, Phys. Rev. E 76, 061107 (2007).

${ }^{34}$ G. Ganzenmüller and P. J. Camp, J. Chem. Phys. 127, 154504 (2007).

${ }^{35}$ M. P. Allen and D. J. Tildesley, Computer Simulation of Liquids (Clarendon, Oxford, 1986).

${ }^{36}$ D. P. Landau and K. Binder, A Guide to Monte Carlo Simulations in Statistical Physics, 2nd ed. (Cambridge University Press, Cambridge, 2005).

${ }^{37}$ E. Lomba, J. J. Weis, N. G. Almarza, F. Bresme, and G. Stell, Phys. Rev. E 49, 5169 (1994).

${ }^{38}$ E. Lomba, C. Martín, and N. G. Almarza, Mol. Phys. 101, 1667 (2003).

${ }^{39}$ A. M. Ferrenberg and D. P. Landau, Phys. Rev. B 44, 5081 (1991).

${ }^{40}$ H. W. J. Blöte, E. Luijten, and J. R. Heringa, J. Phys. A 28, 6289 (1995).

${ }^{41}$ K. Binder, Z. Phys. B: Condens. Matter 43, 119 (1981).

${ }^{42}$ N. B. Wilding, Phys. Rev. E 52, 602 (1995).

${ }^{43}$ M. M. Tsypin and H. W. J. Blötte, Phys. Rev. E 62, 73 (2000).

${ }^{44}$ J. Pérez-Pellitero, P. Ungerer, G. Orkoulas, and A. D. Mackie, J. Chem. Phys. 125, 054515 (2006).

${ }^{45}$ F. Lado, M. Lombardero, E. Enciso, S. Lago, and J. L. F. Abascal, J. Chem. Phys. 85, 2916 (1986); F. Lado, Mol. Phys. 47, 283 (1982); 47, 299 (1982).

${ }^{46}$ G. Pastore, R. Santin, S. Taraphder, and F. Colonna, J. Chem. Phys. 122, 181104 (2005).

${ }^{47}$ P. H. Fries and M. Cosnard, J. Phys. (France) 48, 723 (1987).

${ }^{48}$ E. Enciso, F. Lado, M. Lombardero, J. L. F. Abascal, and S. Lago, J. Chem. Phys. 87, 2249 (1987).

${ }^{49}$ L. Belloni, J. Chem. Phys. 98, 8080 (1993).

${ }^{50}$ J. S. Høye, E. Lomba, and G. Stell, Mol. Phys. 75, 1217 (1992).

${ }^{51}$ E. Lomba, Mol. Phys. 68, 87 (1989).

${ }^{52}$ A. G. Schlijper, M. M. Telo da Gama, and P. G. Ferreira, J. Chem. Phys. 98, 1534 (1993). 